\title{
Antidepressant-Like Effect of Bauhinia blakeana Dunn in a Neuroinflammation Model in Mice
}

\author{
Maribel Herrera-Ruiz Mayra A. Santillán-Urquiza Ofelia Romero-Cerecero \\ Alejandro Zamilpa Enrique Jiménez-Ferrer Jaime Tortoriello \\ Centro de Investigación Biomédica del Sur, Instituto Mexicano del Seguro Social, Morelos, Mexico
}

\section{Significance of the Study}

- The objective of this work was to evaluate the antidepressant effect of Bauhinia blakeana on lipopolysaccharide-induced neuroinflammation in mice.

- Purified fractions from Bauhinia blakeana demonstrated antidepressant effect and counteracted the neuroinflammation.

- Bauhinia blakeana exerts immunomodulatory effect.

- Rutin, a glycosylated flavonoid, was identified as the main compound.

\section{Keywords}

Rutin · Antidepressant · Lipopolysaccharides •

Neuroinflammation - Cytokines · Medicinal plants .

Mexican traditional medicine

\begin{abstract}
Objective: To evaluate the antidepressant effect of Bauhinia blakeana and a standardized fraction in the forced swimming test (FST) on mice with neuroinflammation induced with lipopolysaccharides (LPS). Materials and Methods: Evaluation of the antidepressant effect of Bauhinia blakeana hydroalcoholic extract (BbHA) and its fractions was carried out in behavioral tests on mice with LPS-induced neuroinflammation. Results: BbHA had a significant antidepressant effect, measured on healthy mice in the FST. Bio-guided chemical separation of the extract produced a methanolic fraction (BbMe), which decreased the immobility time in FST. In this test, the intraperitoneal administration of LPS induced de-
\end{abstract}

\begin{tabular}{ll}
\hline KARGER & $\begin{array}{l}\text { (c) } 2019 \text { The Author(s) } \\
\text { Published by S. Karger AG, Basel }\end{array}$ \\
karger@karger.com & This is an Open Access article licensed under the Creative Commons \\
www.karger.com/mpp & $\begin{array}{l}\text { Attribution-NonCommercial-4.0 International License (CC BY-NC) } \\
\text { (http://www.karger.com/Services/OpenAccessicense), applicable to } \\
\text { the online version of the article only. Usage and distribution for com- } \\
\text { mercial purposes requires written permission. }\end{array}$
\end{tabular}

pression in mice, and $\mathrm{BbHA}$ and BbMe counteracted this effect, significantly decreasing the induced depression. Quantification of inflammatory mediators (IL-10, IL-4, IL-6, IL-1 $\beta$, and TNF- $\alpha$ ) in the brain demonstrated that BbHA and BbMe effectively decreased the effect of LPS on the brain concentration of all measured cytokines. Conclusions: Bauhinia blakeana produced an antidepressant effect, while BbMe also exerted a modulating effect, on the damage induced by LPS. Rutin, a glycosylated flavonoid, was identified as the main compound in the active fraction, which could mediate in the antidepressant and immunomodulatory effect.

\section{C) 2019 The Author(s)}

Published by S. Karger AG, Basel

\section{Introduction}

Depression affects 350 million persons and is now starting at young ages, affecting working people, and has become the leading cause of disability in terms of total work

Dr. Jaime Tortoriello

Centro de Investigación Biomédica del Sur

Instituto Mexicano del Seguro Social

Argentina No. 1, Xochitepec, Morelos 62790 (Mexico)

E-Mail jtortora2@yahoo.es 
years lost due to this condition [1]. It is estimated that in 2030, depression will be the second cause of morbidity in middle-income countries and the third in the low-income countries [2]. Drugs for its treatment present serious adverse effects, such as dry mouth, blurred vision, constipation, postural hypotension, arrhythmias, and weight gain [3]. Species from Bauhinia genus are used in Mexican traditional medicine for treating dysentery, diarrhea, colds, diabetes, infections of the urinary tract, and as anti-inflammatories $[4,5]$. But, in some areas, the aerial parts are especially used for treating "tiricia," a term employed in traditional medicine to refer to a disorder consisting of states of loss of appetite, lack of motivation, and paleness, occurring in persons with sadness, disappointment, and bad mood [4]. Bauhinia blakeana Dunn, from the Fabaceae family [6], has been identified as one of the species utilized for its empirical properties for the treatment of depression.

In the present work, we evaluated the antidepressant effect of the extract from Bauhinia blakeana and a standardized fraction in the forced swimming test (FST) on mice with lipopolysaccharide (LPS)-induced neuroinflammation.

\section{Materials and Methods}

\section{Plant Material}

Leaves of Bauhinia blakeana Dunn were collected in Juitepec, Morelos, Mexico. A voucher specimen was analyzed by M.Sc. Santiago Xolalpa, IMSSM Herbarium Taxonomist, which was safeguarded under registration number IMSSM-16408. Plant material was dried and afterward was ground until obtaining particles of $<5 \mathrm{~mm}$. Two kilograms were extracted by maceration in an alcoholic solution (ethanol 60\%) at room temperature for $2 \mathrm{~h}$ (repeated 3 times). The B. blakeana hydroalcoholic extract (BbHA, $156 \mathrm{~g}$ ) was concentrated with a rotatory evaporator (Heidolph Laborota 4000 , Germany). In order to eliminate all residues of solvent, the extract was lyophilized, weighed $(7.8 \%$ yield $)$, and stored at $<4^{\circ} \mathrm{C}$ until its use for pharmacological tests.

\section{Chemical Separation of Fractions}

BbHA extract was separated by bipartition: $100 \mathrm{~g}$ of BbHA was dissolved in $500 \mathrm{~mL}$ of water and $500 \mathrm{~mL}$ of ethyl acetate. Phase separation allowed the obtaining of an aqueous fraction (BbAq, 91.9\%) and the ethyl acetate fraction (BbAt, 8.1\%). The dried BbAq fraction was suspended in a solution of acetone/methanol 80:20 (50 $\mathrm{mL}$ ), obtaining an acetone-soluble fraction (BbAc) with a $65.3 \%$ yield. Finally, the precipitated portion was suspended in methanol $(500 \mathrm{~mL})$ and soluble phase was concentrated by low-pressure distillation, obtaining a methanolic fraction (BbMe) with a $19.5 \%$ yield.

\section{Chemical Characterization of the BbMe Fraction}

In order to identify the bioactive compounds, the most active fraction $(\mathrm{BbMe})$ was subjected to chromatographic analysis using an Acquity UPLC (Waters, Milford, MA, USA) provided by quaternary pump, autosampler, column oven, and a photodiode array-detector. Chromatographic separations were performed employing an Acquity UPLC BEH $1.7 \mathrm{~m}-\mathrm{C} 18$ column at a flow rate of $0.4 \mathrm{~mL} / \mathrm{min}$. The column was eluted with $0.1 \%$ formic acid in water (A) and with $0.1 \%$ formic acid in acetonitrile (B). The column was maintained at $100 \%$ of $A$ for 1 min and subsequently ramped to $100 \%$ of $B$ (curve 6 ) over $11 \mathrm{~min}$, followed by a 4 -min period at $100 \%$ of $\mathrm{B}$, prior to a rapid return to $100 \%$ of $\mathrm{A}$ and an equilibration period of $2 \mathrm{~min}$. The column was maintained at temperatures of $40^{\circ} \mathrm{C}$. Injection volume was $5 \mu \mathrm{L}$ and absorbance was measured at a wavelength of $350 \mathrm{~nm}$. Rutin was identified by direct comparison of retention time and UV spectra and positive molecular ion $(\mathrm{M}+1)$ with those of the reference standard (R5143; Sigma-Aldrich, St. Louis, MO, USA; Fig. 4).

The amount of rutin was estimated by interpolation of peak areas and comparison with a calibration curve developed for this flavonol. The calibration curve was linear in the range of 1.25-40 $\mu \mathrm{g} / \mathrm{mL}$ of rutin $(1.25,2.5,5,10,20$, and $40 \mu \mathrm{g} / \mathrm{mL} ; y=124,046 x+$ 253,$\left.625 ; R^{2}=0.999\right)$. Analyses were repeated in triplicate. Results are expressed as mean values in mg of rutin/g of dry extract.

\section{Animals}

Male ICR mice (30-35 g, from the ENVIGO RMS Company) were organized in groups of 7 animals under controlled conditions (at least 3 weeks before experimentation) with a 12-h dark-light cycle at a temperature of $20 \pm 1^{\circ} \mathrm{C}$, with free access to food (Labdiet 5008; Brentwood, MO, USA) and water. Behavioral tests were carried out in a sleepy buffered room illuminated with a red light and with the aid of a video recording system.

\section{Experimental Design}

The study was divided into 2 phases. The first consisted of the acute evaluation of different treatments of Bauhinia blakeana, including BbHA (100, 250, and $500 \mathrm{mg} / \mathrm{kg}$, p.o.), BbAt $(500 \mathrm{mg} / \mathrm{kg}$, p.o.), BbAc (5 mg/kg, p.o.), and BbMe (5, 25, and $200 \mathrm{mg} / \mathrm{kg}$, p.o.), in the FST. Imipramine (IMI, $15 \mathrm{mg} / \mathrm{kg}$ ) was used to treat the positive control group and was administered $30 \mathrm{~min}$ before the test. The negative control group was administered 1\% Tween 20 (VEH; $n=7)$. In order to evaluate whether any of the treatments induced changes in the spontaneous motor activity of the mice, another group of animals were evaluated by means of the open field test (OFT). All treatments were dissolved in 1\% Tween 20 and administered in a volume of $100 \mu \mathrm{L} / 10 \mathrm{~g}$. VEH was also administered to the negative control group. The second phase consisted of mice with LPS-induced neuroinflammation. To this purpose, mice $(n=$ 10 in each group) were administered for 4 days with a daily dose of $0.083 \mathrm{mg} / \mathrm{kg}$ (i.p.) of a fresh solution of LPS (in sterile water) [7]. On the fourth day, in addition to LPS, all of the animals also received the experimental treatments comprising BbHA $(500 \mathrm{mg} / \mathrm{kg}$, p.o.) and $\mathrm{BbMe}$ ( 5 and $25 \mathrm{mg} / \mathrm{kg}$, p.o.). The positive control group was treated with IMI (15 mg/kg, i.p.), while the negative control group received vehicle ( $1 \%$ Tween $20, \mathrm{VEH})$, an anti-inflammatory control group received meloxicam (MEL, $1.0 \mathrm{mg} / \mathrm{kg}$, p.o.), and an antibiotic group, received minocycline (MIN, $50 \mathrm{mg} / \mathrm{kg}$, p.o.). A group treated with saline solution (SS) without LPS was included. During the following 4 days, the animals were administered only the respective treatments. On day 8 , all of the mice were submitted to the FST (without previous training, modified method of Porsolt) and OFT. Afterward, the animals were sacrificed in order to obtain the brains, from which cytokine concentrations were quantified; 5 pools were prepared, each with 2 mice. 
Forced Swimming Test

With an experimental procedure widely used to evaluate a possible antidepressive effect, we proceeded with pre-test training during which the mice were placed for $15 \mathrm{~min}$ individually in glass containers with water at a depth of $15 \mathrm{~cm}$ and at a temperature of $25 \pm 2{ }^{\circ} \mathrm{C}$. Subsequently, different treatments were given to each group of animals. Test evaluation time was of $5 \mathrm{~min}$, and the immobility time was measured.

\section{Open Field Test}

An open field device was used, which was made with clear acrylic walls $(30 \times 30 \times 15 \mathrm{~cm})$ and a black floor (with 9 marked divisions). Each animal was placed at the center of the field and then the following parameters were evaluated for $5 \mathrm{~min}$ : total crossings (TC) and rearings (R). This test is widely employed to evaluate the spontaneous motor activity of the rodent in a new environment.

\section{Quantification of Cytokines}

The brain pools were homogenized with an electric device in a phosphate buffer solution at $\mathrm{pH}=8$, also containing $0.1 \%$ phenylmethylsulfonyl fluoride (protease inhibitor). Once homogenized, the tissue was centrifuged at 12,000 rpm for $5 \mathrm{~min}$. Cytokine levels were estimated in the supernatants ( $n=5$ per pool for each group), for which it was also necessary to measure the total protein concentration of the supernatant, using the Bradford spectrophotometric method. Albumin was used to construct a standard calibration curve.

Quantification of each cytokine was carried out by the ELISA method using cytokine kits (OptEIATM ELISA sets; BD Biosciences, Franklin Lakes, NJ, USA) and following the manufacturer's instructions. For TNF- $\alpha$, IL-6, IL-4, and IL-10, $100 \mu \mathrm{L} /$ well of detection antibody was added, as well as streptavidin-horseradish peroxidase enzyme. For IL-1 $\beta, 100 \mu \mathrm{L} /$ well of detection antibody was added and streptavidin-horseradish peroxidase enzyme (100 $\mu \mathrm{L} /$ well) was used. All were incubated for $1 \mathrm{~h}$ and then washed with $300 \mu \mathrm{L} /$ well 5 times with a phosphate buffer solution (added with $0.05 \%$ of Tween 20 ).

\section{Statistical Analysis}

Statistical analysis was performed with an SPSS version 17.0 software program (SPSS, Inc., released 2008; SPSS Statistics for Windows, version 17.0; SPSS, Inc., Chicago, IL, USA) and the results were expressed as mean $\pm \mathrm{SD}$. Data were analyzed by using analysis of variance (ANOVA) followed by the Dunnett post hoc test $(p<0.05)$.

\section{Results}

\section{Effect of BbHA Extract and Derived Fractions on FST} and $O F T$

In the FST, IMI decreased immobility time by 49.53\%. Similarly, BbHA (500 mg/kg) also achieved a significant decrease of immobility of up to $69.01 \%$ in comparison with the negative control group (VEH). In this trial, administration of BbHA (100 and $250 \mathrm{mg} / \mathrm{kg}$ )
Table 1. Effects produced by different treatments from Bauhinia blakeana on mice subjected to the forced swimming test and the open field test

\begin{tabular}{lccc}
\hline $\begin{array}{l}\text { Treatment, } \\
\text { mg/kg }\end{array}$ & Immobility, s & $\begin{array}{l}\text { TC, number } \\
\text { of events }\end{array}$ & $\begin{array}{l}\text { R, number } \\
\text { of events }\end{array}$ \\
\hline VEH & $237.4 \pm 21.72$ & $150.00 \pm 25.33$ & $67.2 \pm 7.40$ \\
IMI 15 & $117.66 \pm 24.38^{*}$ & $151.83 \pm 8.57$ & $56.6 \pm 8.88$ \\
BbHA 100 & $228.85 \pm 27.06$ & - & - \\
BbHA 250 & $194.5 \pm 24.03$ & - & - \\
BbHA 500 & $163.83 \pm 26.01^{*}$ & $67.6 \pm 17.36^{*}$ & $39.4 \pm 13.79^{*}$ \\
BbAt 500 & $214.4 \pm 16.81$ & - & - \\
BbAc 5 & $239.80 \pm 24.86$ & - & - \\
BbMe 5 & $147.50 \pm 18.69^{*}$ & $79.57 \pm 11.46^{*}$ & $50.6 \pm 13.63^{*}$ \\
BbMe 25 & $150.00 \pm 19.37^{*}$ & $87.00 \pm 10.99^{*}$ & $43.67 \pm 11.91^{*}$ \\
BbMe 200 & $190.83 \pm 16.03^{*}$ & $71.50 \pm 6.86^{*}$ & $18.6 \pm 6.91^{*}$ \\
\hline
\end{tabular}

IMI, imipramine; BbHA, Bauhinia blakeana hydroalcoholic extract; BbAt, ethyl acetate fraction; BbAc, acetone-soluble fraction; $\mathrm{BbMe}$, methanol-soluble precipitate; $\mathrm{VEH}$, vehicle; -, not evaluated. TC, total crossings $(\mathrm{FD}=6, F[40.8] 2.55, p=0.0001)$; , rearings $(\mathrm{FD}=6, F[13.9] 2.58 ; p=0.0001)$, immobility $(\mathrm{FD}=6$; $F[35.6] 2.04 ; p=0.0001)$. Results are means \pm SD. ${ }^{*} p<0.05$ in comparison with VEH, by means of ANOVA and Dunnett posttest $(n=7)$.

did not achieve a statistically significant effect (Table 1). Fractions BbAt and BbAc (500 mg/kg) did not modify immobility time in the FST, while 5,25 , and $200 \mathrm{mg} / \mathrm{kg}$ of the $\mathrm{BbMe}$ fraction achieved a significant and consistent diminution of immobility. In the OFT, the BbHA fraction $(500 \mathrm{mg} / \mathrm{kg})$ and the BbMe fraction $(5,25$, and $200 \mathrm{mg} / \mathrm{kg}$ ) produced a significant decrease of the TC and $\mathrm{R}$ parameters, in comparison with the $\mathrm{VEH}$ group (Table 1). IMI did not generate changes in these parameters.

\section{Effect of BbHA and BbMe on LPS-Induced Neuroinflammation on FST and OFT}

Animals from the SS group (without LPS), exposed to the FST, exhibited a 240 -s immobility time and, compared to the negative control group (VEH, with LPS), significantly increased immobility time in the FST (Fig. 1). Furthermore, IMI, BbHA (500 mg/kg), and BbMe (5 mg/ $\mathrm{kg}$ ) counteracted the effects of LPS-induced neuroinflammation in the mice, as they significantly decreased immobility time when compared to the VEH and SS groups. In the OFT, administration of IMI, MEL, BbHA (500 mg/ $\mathrm{kg}$ ), and $\mathrm{BbMe}$ ( 5 and $25 \mathrm{mg} / \mathrm{kg}$ ) induced a significant increase in the TC and R parameters when compared to the VEH group (Fig. 2). 
Fig. 1. Effect produced by oral administration of the hydroalcoholic extract of Bauhinia blakeana (BbHA) and the methanolic fraction (BbMe) in ICR mice with LPS-induced neuroinflammation on the FST. Results are means \pm SD. ${ }^{*} p<0.05$ in comparison with $\mathrm{VEH} ;{ }^{\&} p<0.05$ in comparison with the SS group by means of ANOVA and Dunnett post-test $(n=10$; $\mathrm{FD}=9 ; F[26.4] 2.1 ; p=0.0001)$. IMI, imipramine; MIN, minocycline; MEL, meloxicam; VEH, vehicle.

Fig. 2. Effect produced by the oral administration of the hydroalcoholic extract of Bauhinia blakeana (BbHA) and the methanolic fraction (BbMe) in ICR mice with LPS-induced neuroinflammation on the OFT (FD = 9; F[26.4] 2.1; $p=0.0001)$. R, rearings $(\mathrm{FD}=6 ; F[14.9] 2.09 ; p=0.0001)$. Results are means \pm SD. ${ }^{*} p<0.05$ in comparison with $\mathrm{VEH} ;{ }^{*} p<0.05$ in comparison with the SS group by means of ANOVA and the Dunnett post-test $(n=10)$. IMI, imipramine; MIN, minocycline; MEL, meloxicam; $\mathrm{VEH}$, vehicle; TC, total crossings.
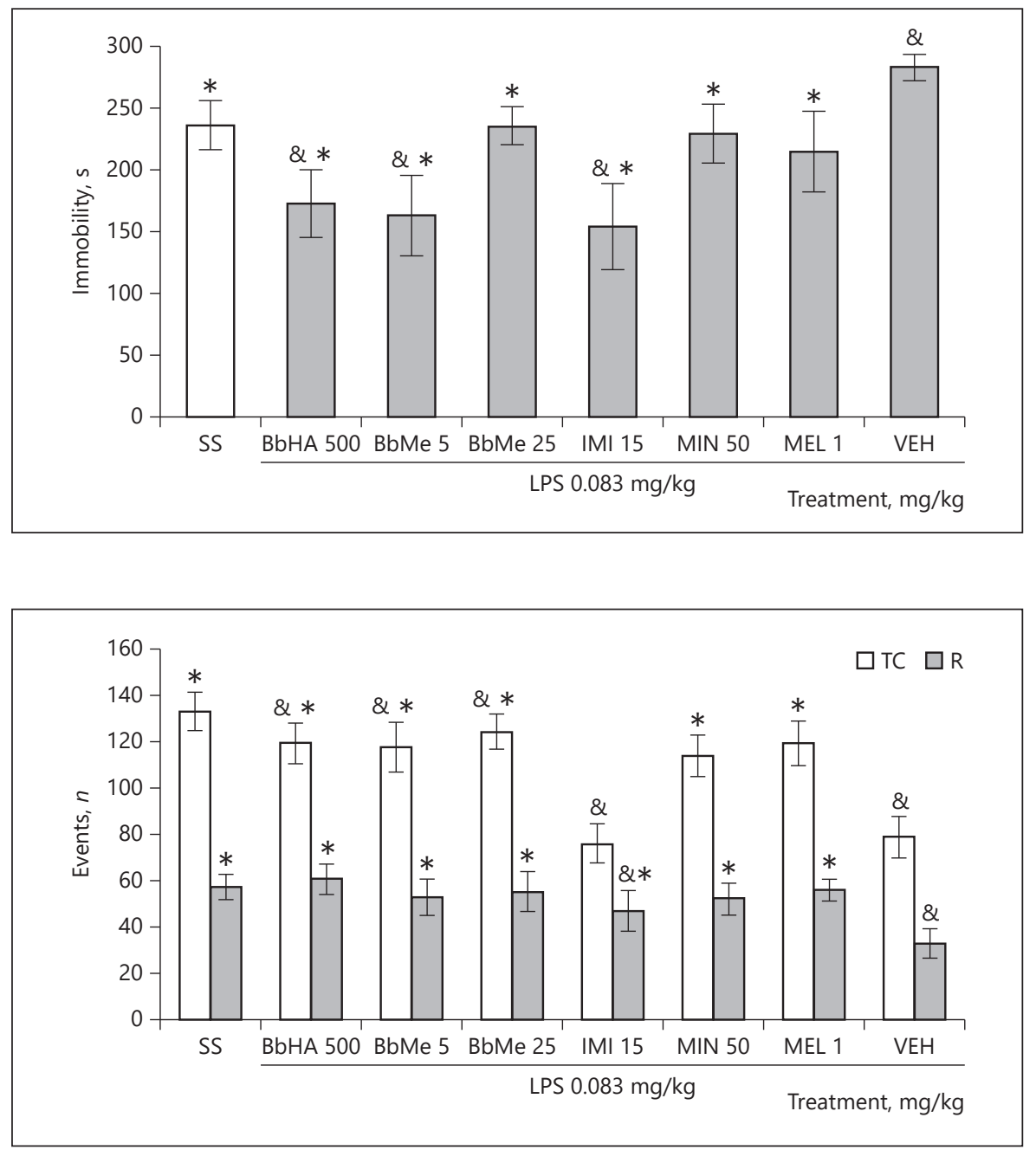

\section{Effect of BbHA Extract and BbMe on Brain Cytokine Levels}

As depicted in Figure 3a, the levels of IL-1 $\beta$ (pg/mg) in the brain homogenate of mice with LPS-induced neuroinflammation were significantly higher than those obtained for the SS group without LPS. The animals administered with BbHA, MIN, MEL, and BbMe demonstrated that IL-1 $\beta$ levels in the brain were significantly lower than those observed in the VEH group, while the groups with IMI and BbMe did not exhibit significant changes when compared to the VEH group.

The IL- 4 concentration was significantly increased in the brain homogenates of the mice pretreated with LPS (see VEH group) when compared to those in the SS group. $\mathrm{BbHA}, \mathrm{MIN}, \mathrm{MEL}$, and $\mathrm{BbMe}$ were able to counteract the effect induced by LPS. BbHA and BbMe reached IL-4 values similar to those of the animals without neuroinflammation from the SS group (Fig. 3b).
IL-6 levels in mice pretreated with LPS (VEH) were significantly higher than those of the SS group. Administration of BbHA, MIN, MEL, and BbMe also counteracted the effect of LPS and produced a significant decrease in IL-6 compared to the group with neuroinflammation (VEH). These results were not different from those of the SS group. IMI and BbMe did not show a significant change compared to the VEH group, although the levels of IL-6 did increase when compared to the SS group (Fig. 3c).

IL-10 levels were significantly increased in the brains of the animals with LPS when compared to those of the SS group. BbHA, MIN, BbMe, and the antidepressant IMI were able to counteract the effect induced by LPS. MEL and $\mathrm{BbMe}$ did not reveal any statistically significant changes when compared to the VEH group. However, we observed a significant increase in IL-10 levels when compared to the SS group (Fig. 3d). 
Fig. 3. Effect of the hydroalcoholic extract of Bauhinia blakeana (BbHA) and the methanolic fraction (BbMe) on the concentration of cytokines in brain of ICR mice with LPS-induced neuroinflammation. ANOVA and Dunnett post-test $(n=$ $10 \pm$ SD). $\mathbf{a}^{\&} p<0.05$ IL- $1 \beta$ vs. SS; ${ }^{*} p<0.05$ IL- $1 \beta$ vs. VEH $(\mathrm{FD}=9 ; F[38.1] 2.44 ; p=$ $0.0001) . \mathbf{b}^{\&} p<0.05$ IL-4 vs. SS; ${ }^{*} p<0.05$ IL-4 vs. VEH $(\mathrm{FD}=9 ; F[20.2] 2.44 ; p=$ $0.0001)$. c $^{\&} p<0.05$ IL-6 vs. SS; ${ }^{*} p<0.05$ IL-6 vs. VEH (FD = 9; $F[16.09] 2.44 ; p=$ $0.0001) . \mathbf{d}^{\&} p<0.05$ IL-10 vs. SS; ${ }^{*} p<0.05$ IL-10 vs. VEH (FD = 9; $F[30.4] 2.44 ; p=$ $0.0001) . \mathbf{e}^{\&} p<0.05$ TNF- $\alpha$ vs. SS; ${ }^{*} p<0.05$ TNF- $\alpha$ vs. VEH (FD = 9; $F[38.03] 2.44 ; p=$ $0.0001)$. IMI, imipramine; MIN, minocycline; MEL, meloxicam; VEH, vehicle.
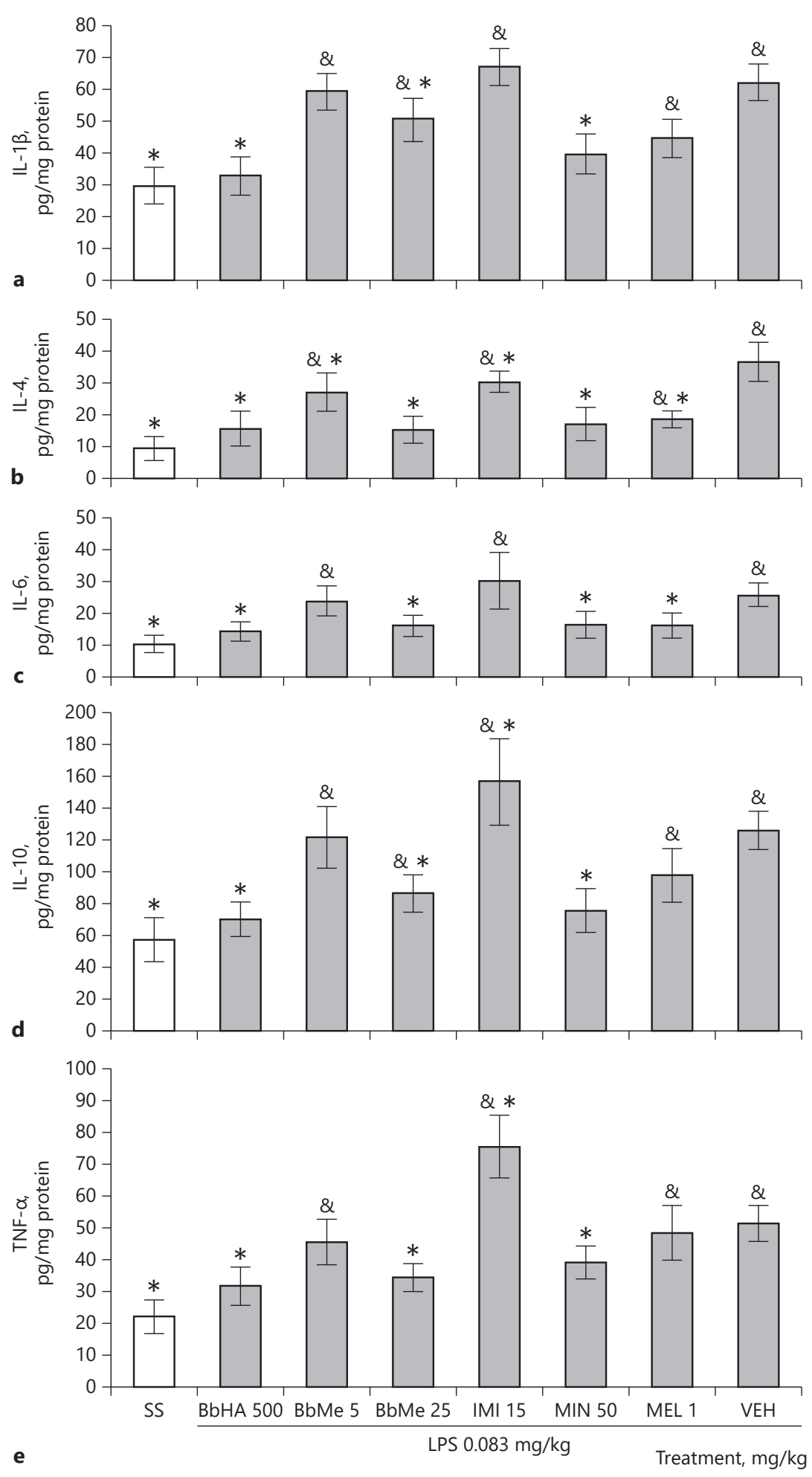


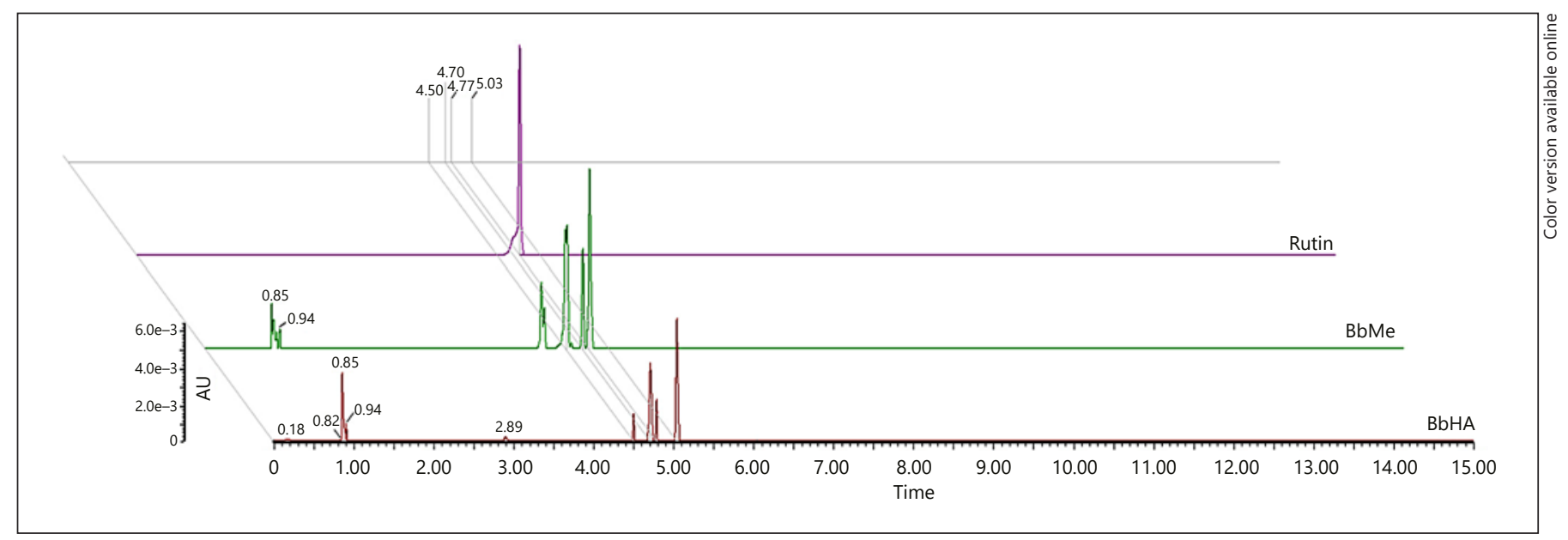

Fig. 4. Chromatographic profile of the hydroalcoholic extract of Bauhinia blakeana (BbHA), the methanol fraction (BbMe), and rutin (4.77 $\mathrm{min} ; \lambda=211,255,355 \mathrm{~nm})$.

The concentration of TNF- $\alpha$ in the brain of mice with LPS-induced neuroinflammation was significantly higher than that of mice without LPS. BbHA, MIN, IMI, and BbMe were able to block the effects of LPS on the concentration of this cytokine, while the groups receiving MEL and $\mathrm{BbMe}$ did not show significant changes when compared to the VEH group (Fig. 3e).

\section{Chemical Characterization of the BbMe Fraction}

Chromatographic profile of BbMe showed the presence of rutin (4.77 min; $\lambda=211,255,355 \mathrm{~nm}$; Fig. 4). An UPLC method was employed, which determined that $\mathrm{BbHA}$ and BbMe contained 0.079 and $5.53 \mathrm{mg} / \mathrm{g}$, respectively, of rutin.

\section{Discussion}

In the present work, Bauhinia blakeana induced a significant decrease of immobility time in healthy ICR mice in the FST, which is widely used for preliminary evaluation of antidepressant activity $[8,9]$. In this test, when the dose of BbMe was increased, antidepressant behavior was reduced; however, at low dosages, the activity was still preserved. There are other studies that have shown that medicinal plants display similar behavior, such as Heteropterys brachiata, which at the highest dose $(750 \mathrm{mg} / \mathrm{kg})$ induced an increase in immobility time in the FST, whereas at the lower dosages, this parameter decreased, indicating antidepressant behavior [7]. B. blakeana decreased locomotion parameters in the OFT (BbHA and BbMe 5,
$25,200 \mathrm{mg} / \mathrm{kg}$ ), indicating first a sedative effect and then showing that the antidepressant behavior is not associated with a motor-stimulating effect.

It has been demonstrated that inflammation plays a crucial role in psychiatric disorders related to stress, such as depression. In addition, major depressive events and their symptoms in patients with IFN- $\gamma$ immunotherapy provide a framework that supports an inflammatory background for this psychiatric disease $[10,11]$. In the present work, LPS induced a significant increase in the immobility time of FST in comparison with healthy mice, and IMI was able to inhibit that effect. In another study, it was observed that this antidepressant $(10 \mathrm{mg} / \mathrm{kg})$, either before or after LPS, in Swiss albino mice, was also capable of decreasing immobility time in FST [12]. LPS induced a significant decrease in the locomotion parameters measured in the OFT and caused a decrease in mouse body weight, both of which were attenuated by IMI. Nevertheless, the animals did not recover completely when compared with healthy animals [13], while MIN (an antibiotic with anti-inflammatory effects) administered to mice with LPS over a period of 4 days attenuated the depressive activity and also the changes of spontaneous motor activity induced by LPS [14]. In the present work, we observed that MEL (a non-selective inhibitor of cyclooxygenase enzymes) counteracted the behavioral effects induced by LPS in both the FST and the OFT. This substance has been reported to exert antidepressant effects and to counteract the toxic effects caused by the administration of LPS in various biological models. BbHA and BbMe resulted in significant antidepressant activity. It is notewor- 
thy that the higher dose of BbMe had an effect which was lower than that of the $5 \mathrm{mg} / \mathrm{kg}$ dose; however, it was capable of counteracting LPS effects when the animals were evaluated in the OFT. In this test, all treatments derived from Bauhinia blakeana exhibited an increase in motor activity independently of the dose.

We also measured the concentrations of some cytokines, which induce a disease condition characterized by decreased sugar consumption, cognitive impairment, and neurovegetative symptoms, which together represent a phenotype of depression symptoms in humans [15].

LPS increases levels of IL- $\beta$ and TNF- $\alpha$, which work also as neuro-regulators [16]. In this work, LPS gave rise to an increase in cerebral TNF- $\alpha$, IL- 6 , and IL- $1 \beta$ levels, as well as those of the anti-inflammatory levels, such as IL-4 and IL-10 [17]. In the present work, IMI modified the concentrations of IL-10 and TNF- $\alpha$ in the brain and MIN counteracted the elevation of all measured cytokines. It has already been demonstrated that the administration of this drug for 3 consecutive days prior to injection of LPS $(0.33 \mathrm{mg} / \mathrm{kg})$ is capable of reducing the expression of Toll-2 surface receptors (TLR2) on brain microglia, in addition to reducing the mRNA expression of cytokines such as IL- $1 \beta$ and IL-6 [18] and TNF- $\alpha$ [19, $20]$. The results obtained herein showed that MEL was able to avoid elevation in the brain concentration of IL-6 compared to that of the VEH group, while having no effect on IL- $1 \beta$ and TNF- $\alpha$ concentration. MEL did not modify IL- 6 but increased TNF- $\alpha$ in rats with intra-arterial administration of $10 \mu \mathrm{g} / \mathrm{kg}$ of LPS in rats [21], denoting a selective involvement of MEL in the effects of LPS on the different cytokines.

BbHA decreased the effects of the LPS on brain cytokine concentrations, indicating that this treatment acts as a modulator of the general activation process of the immune response. The extract caused mice under an immune-stress condition to return to conditions similar to those of mice without the immune challenge. BbMe decreases the concentration of IL-4, which is produced in response to the activation of $\mathrm{CD} 4$ (or Th2-response) $\mathrm{T}$ lymphocytes. Apparently, at the lower dose of $5 \mathrm{mg} / \mathrm{kg}$ of $\mathrm{BbMe}$, in which an antidepressant effect was observed (in the FST, the healthy as well as those receiving LPS), the effect of BbMe on the cytokines concentration was very precise, affecting only the levels of IL-4, while at the higher dose of $25 \mathrm{mg} / \mathrm{kg}$, the concentration of all cytokines was significantly lower than those of the VEH group. However, at this dose, the BbMe fraction induced a decrease of antidepressant behavior in comparison to that resulting from the lower dose administered to mice treat- ed with LPS. BbMe diminished IL-10 levels. This effect on the regulation of pro-inflammatory cytokines in the brain may not be related to IL-10; therefore, the capacity of this fraction to regulate the production of TNF- $\alpha$, IL- $1 \beta$, and IL-6 in response to the presence of LPS can be either by direct modulation of the cells producing these molecules or by means of a mechanism in which a dynamic participation of different, secondary metabolites in BbMe modulate the associated response of LPS on the concentrations of the anti-inflammatory and pro-inflammatory cytokines.

Our results indicate that Bauhinia blakeana presents an antidepressive effect and acts as an immunomodulator; these effects are not limited to reducing the behavioral alterations caused by the exposure of the animals to a stressful environment such as the FST. It is also able to promote a regulatory effect on the immune response of the animals exposed to LPS, which also modified the behavioral response by increasing immobility time in the FST, a time that was statistically greater than those of the healthy animals. The chemical analysis evidenced the presence of rutin in the BbMe fraction; this compound suppresses TNF- $\alpha$ production in human umbilical cord endothelial cell cultures exposed to LPS [22] and decreases the production of this cytokine in $\mathrm{BALB} / \mathrm{c}$ mice that were stimulated with this pyrogen [23]. Also, rutin possesses a selective antidepressant effect when evaluated in the tail suspension test but not in the FST; this latter effect was only observed when rutin was administered to mice [24]. In addition, it has also been reported that a methanolic extract of the Hypericum perforatum does not present activity in the FST, but the addition of rutin to that extract results in a strong pharmacological effect [25].

\section{Conclusion}

Bauhinia blakeana acts as antidepressant and as a modulator of the immune response upon the damage induced by LPS. Rutin was identified as the main compound in the active fraction, which could mediate in the antidepressant and immunomodulatory effect.

\section{Acknowledgments}

This work was supported by Instituto Mexicano del Seguro Social (FIS-IMSS/PROT/MD13/1261). Mayra Alejandra SantillánUrquiza is grateful for the Master degree fellowship from CONACyT (267252) and the complementary support from IMSS (99185392). 


\section{Statement of Ethics}

All experiments were performed in accordance with the 1986 UK Animals (Scientific Procedures) Act and associated guidelines: EU Directive 2010/63/EU for animal experiments. The experimental protocol was revised and approved by the Institutional Research and Ethics Committee (R-2012-1701-55).

\section{Disclosure Statement}

The authors declare that they do not have any conflict of interest.

\section{References}

1 National Institute of Mental Health (NIMH). Depression 2010. [accessed June 13, 2018]. http://www.nimh.nih.gov/index.shtml.

2 Willner P, Scheel-Krüger J, Belzung C. The neurobiology of depression and antidepressant action. Neurosci Biobehav Rev. 2013 Dec;37(10 Pt 1):2331-71.

3 Uriarte BV. Psicofarmacología. In: Trillas, México, 2009, 6th ed., pp. 153-8.

4 Zolla C. Diccionario enciclopédico de la medicina tradicional mexicana. In: Instituto $\mathrm{Na}$ cional Indigenista. ed. Mexico: Segunda; 1994. pp. 805-6.

5 Willain Filho A, Breviglieri E, Cechinel Filho V, Santos AR. Antinociceptive effect of the hydroalcoholic extract of Bauhinia splendens stems in mice. J Pharm Pharmacol. 1997 Aug; 49(8):823-7.

6 Mak CY, Cheung KS, Yip PY, Kwan HS. Molecular evidence for the hybrid origin of Bauhinia blakeana (Caesalpinioideae). J Integr Plant Biol. 2008 Jan;50(1):111-8.

7 Zhang ZJ. Therapeutic effects of herbal extracts and constituents in animal models of psychiatric disorders. Life Sci. 2004 Aug; 75(14):1659-99.

8 Petit-Demouliere B, Chenu F, Bourin M. Forced swimming test in mice: a review of antidepressant activity. Psychopharmacology (Berl). 2005 Jan;177(3):245-55.

9 Huerta-Reyes M, Herrera-Ruiz M, GonzálezCortazar M, Zamilpa A, León E, Reyes-Chilpa $R$, et al. Neuropharmacological in vivo effects and phytochemical profile of the extract from the aerial parts of Heteropterys brachiata (L.) DC. (Malpighiaceae). J Ethnopharmacol. 2013 Mar;146(1):311-7.

10 Khansari PS, Sperlagh B. Inflammation in neurological and psychiatric diseases. Inflammopharmacology. 2012 Jun;20(3):103-7.
11 Felger JC, Lotrich FE. Inflammatory cytokines in depression: neurobiological mechanisms and therapeutic implications. Neuroscience. 2013 Aug;246:199-229.

12 Mello BS, Monte AS, McIntyre RS, Soczynska JK, Custódio CS, Cordeiro RC, et al. Effects of doxycycline on depressive-like behavior in mice after lipopolysaccharide (LPS) administration. J Psychiatr Res. 2013 Oct;47(10): 1521-9.

13 Cohn DW, Kinoshita D, Palermo-Neto J. Antidepressants prevent hierarchy destabilization induced by lipopolysaccharide administration in mice: a neurobiological approach to depression. Ann N Y Acad Sci. 2012 Jul; 1262(1):67-73.

14 Henry CJ, Huang Y, Wynne A, Hanke M, Himler J, Bailey MT, et al. Minocycline attenuates lipopolysaccharide (LPS)-induced neuroinflammation, sickness behavior, and anhedonia. J Neuroinflammation. 2008 May;5(1): 15.

15 Dantzer R, O'Connor JC, Freund GG, Johnson RW, Kelley KW. From inflammation to sickness and depression: when the immune system subjugates the brain. Nat Rev Neurosci. 2008 Jan;9(1):46-56.

16 Felger JC, Alagbe O, Hu F, Mook D, Freeman AA, Sanchez MM, et al. Effects of interferonalpha on rhesus monkeys: a nonhuman primate model of cytokine-induced depression. Biol Psychiatry. 2007 Dec;62(11):1324-33.

17 Layé S, Parnet P, Goujon E, Dantzer R. Peripheral administration of lipopolysaccharide induces the expression of cytokine transcripts in the brain and pituitary of mice. Brain Res Mol Brain Res. 1994 Nov;27(1):157-62.

18 Soczynska JK, Mansur RB, Brietzke E, Swardfager W, Kennedy SH, Woldeyohannes $\mathrm{HO}$, et al. Novel therapeutic targets in depression: minocycline as a candidate treatment. Behav Brain Res. 2012 Dec;235(2):302-17.
19 Liu D, Yang PS. Minocycline hydrochloride nanoliposomes inhibit the production of TNF- $\alpha$ in LPS-stimulated macrophages. Int J Nanomedicine. 2012;7:4769-75.

20 Roth J, Hübschle T, Pehl U, Ross G, Gerstberger R. Influence of systemic treatment with cyclooxygenase inhibitors on lipopolysaccharide-induced fever and circulating levels of cytokines and cortisol in guinea-pigs. Pflugers Arch. 2002 Jan;443(3):411-7.

21 Sutcigil L, Oktenli C, Musabak U, Bozkurt A, Cansever A, Uzun O, et al. Pro- and anti-inflammatory cytokine balance in major depression: effect of sertraline therapy. Clin Dev Immunol. 2007;2007:76396.

22 Guruvayoorappan C, Kuttan G. Rutin inhibits nitric oxide and tumor necrosis factor-alpha production in lipopolysaccharide and concanavalin-a stimulated macrophages. Drug Metabol Drug Interact. 2007;22(4):26378.

23 Machado DG, Bettio LE, Cunha MP, Santos AR, Pizzolatti MG, Brighente IM, et al. Antidepressant-like effect of rutin isolated from the ethanolic extract from Schinus molle L. in mice: evidence for the involvement of the serotonergic and noradrenergic systems. Eur J Pharmacol. 2008 Jun;587(13):163-8

24 Nöldner M, Schötz K. Rutin is essential for the antidepressant activity of Hypericum perforatum extracts in the forced swimming test. Planta Med. 2002 Jul;68(7):577-80.

25 Xu PX, Wang SW, Yu XL, Su YJ, Wang T, Zhou WW, et al. Rutin improves spatial memory in Alzheimer's disease transgenic mice by reducing $A \beta$ oligomer level and attenuating oxidative stress and neuroinflammation. Behav Brain Res. 2014 May;264:17380. 